\title{
PROBLEMAS PROPUESTOS
}

Con el afán de promover el diálogo matemático, entre los cultores y amantes de esta disciplina, la Sección de Algebra del Departamento de Matemáticas de la Universidad del Norte, presentó en la edición anterior, tres problemas, cuyas soluciones presentamos en esta edición.

Para continuar este diálogo, la Sección de Estadística de nuestro Departamento, presenta para su discusión y análisis dos problemas de probabilidades. Le invitamos a comunicarnos la solución que Ud. encuentra para estos problemas.

PRIMER PROBLEMA.

1. Una caja contiene $\underline{a}$ esferas blancas $y \underline{b}$ esferas negras. De ellas se extrae al azar $\mathrm{N}$ esferas, simultáneamente. Bajo la hipótesis que todas las esferas 
tienen la misma probabilidad de ser elegidas, determine para un valor de $\mathrm{N}$ fijo, cuál es el valor de $\mathrm{p}$ a, que hace máxima la probabilidad de que las $N$ esferas extraídas $\mathrm{p}$ sean blancas.

SEGUNDO PROBLEMA.

2. ¿Cuál es la probabilidad de que un entero escogido al azar entre los $\mathrm{N}$ primeros enteros, sea divisible al menos por dos de los números primos 2,3,5,7?

Indicación: Suponga $\mathrm{N}$ lo suficientemente grande de modo que $1 / \mathrm{N}$ sea no significativo en los resultados finales.

SOLUCIONES PROBLEMAS DE ALGEBRA.

PRIMER PROBLEMA.

Sean $a, b, c$ enteros distintos. Demuestre que no existe un polinomio $p(x)$ con coeficientes enteros tal que:

i) E1 resto de dividir $p(x)$ por $(x-a)$ es $b$

ii) E1 resto de dividir $p(x)$ por $(x-b)$ es $c$

iii) El resto de dividir $p(x)$ por $(x-c)$ es $a$

Solución: Supongamos que existe $p(x)=\sum_{i=0}^{n} a_{i} x^{i}$ con $a_{i} \varepsilon \mathrm{z}$ satisfaciendo las propiedades (i), (ii), (iii). Ya que el resto de dividir $p(x)$ por $p(x-\alpha)$ es $p(\alpha)$, tenemos que $p(a)=b=\sum_{i=o}^{n} a_{i} a^{i} ; \quad p(b)=c=\sum_{i=0}^{n} a_{i} b^{i} \quad$ y $\quad p(c)=a=\sum_{i=0}^{n} a_{i} c^{1} \alpha$, luego: $b-c=\sum_{i=1}^{n} a_{i}\left(a^{i}-b^{i}\right) ; c-a=\sum_{i=1}^{n} a_{i}\left(b^{i}-c^{i}\right) \mathrm{y} a-b=\sum_{i=1}^{n} a_{i}\left(c^{i}-a^{i}\right)$

Recordando que $\alpha^{n}-\beta^{n}$ es divisible por $\alpha-\beta$ para cualquier $n$ y todo $\alpha \neq \beta$, sabemos que existen enteros $A$, B y C tales que: $b-c=(a-b)$ A; $c-a=(b-c)$ B y $a-b=(c-a)$ C. 
Entonces $(b-c)(c-a)(a-b)(1-A B C)=0 \quad \mathrm{y}$ siendo $a, b, c$ distintos concluímos que $A B C=1$, y como $A, B, C$ son enteros entonces $A, B, C \varepsilon\{-1,+1\}$. Es imposible que $A, B$ ó $C$ sea -1 pues se contradice el hecho que $a, b, c$ sean distintos. Si $A, B y C$ son positivos, tenemos que $b-c=a-b$ y $c-a=b-c$ de donde $b=c$ que es una contradicción.

Por lo tanto no existe tal polinomio.

SEGUNDO PROBLEMA.

Encontrar todos los pares de enteros no negativos tales que su suma sea un divisor de su producto.

Solución: Sea $(x, y)$ una solución, entonces $(y, x)$ también es solución, luego sólo calcularemos aquellos $(x, y)$ tales que $o \leq x \leq y$.

a) Es claro que $(o, x)$ es solución para todo $x \in \mathrm{IN}$

b) Sean $x \neq o \neq y, y=x+\lambda$ con $\lambda \geq o$ entonces:

$$
\begin{aligned}
& (x+y) \mid x y \Leftrightarrow \exists z \varepsilon \mathbb{N} \text { tq } x(x+\lambda)=(x+x+\lambda) z \circ \text { sea } \\
& x^{2}+x \cdot(\lambda-2 z)-\lambda z=0 \text {, luego } x=\frac{2 z-\lambda \pm \sqrt{\lambda^{2}+4 z^{2}}}{2},
\end{aligned}
$$

pero es sabido que $\lambda^{2}+4 \mathrm{z}^{2}$ es un cuadrado perfecto si y sólo si $\lambda=\left(m^{2}-n^{2}\right) k$ y $\mathrm{z}=$ mnk para cualquier $k, m, m \varepsilon \mathbf{Z} t q \lambda \geq o, z>o$. En tal caso tenemos: $(x, y)=k(m+n)(n, m)$ y $(x, y)=k(m-n)(-m, n)$

Como ambas fórmulas recorren los mismos valores al variar $k, m, n \varepsilon \mathbf{Z}$, las soluciones son: $(x, y)=k(m+n)(n, m)$ con $m, n, k \varepsilon \mathbb{N}$ y $m \geq n$.

En consecuencia, si $R=($ ox $\mathbb{N}) \cup\{k(m+n)(n, m) /$ $k, m, n \varepsilon \mathbb{N}, m \geq n\}$ entonces las soluciones al problema es- tán dadas por $R U R^{-1}$ 
TERCER PROBLEMA.

Los coeficientes $C_{m, n}^{(k)}$ se definen mediante $(1+x)^{m}(1-x)^{n}=\sum_{k=0}^{m+n} \quad C_{m, n}^{(k)} x^{k}$ con $m, n \geq o$. Demuestre que $\sum_{k=0}^{m+n}\left(C_{m, n}^{(k)}\right)^{2}=\frac{(2 m) !(2 n) !}{m ! n !(m+n) !}$

Solución: Sea

$A_{n}=\left\{m \varepsilon \mathbb{N} / \sum_{k=o}^{m+n}\left(C_{m, n}^{(k)}\right)^{2}=\frac{(2 m) !(2 n) !}{m ! n !(m+n) !}\right.$ para $n$ fijo $\}$

Basta probar que $A_{n}=\mathbb{N} \forall n \varepsilon \mathbb{N}$. Lo haremos por inducción es $n$.

a) Debemos probar que $A_{o}=\mathbb{N}$. Para ello observemos que $(1+x)^{m}(1-x)^{n}=(1+x)^{m}=\sum_{k=o}^{m}\left(\begin{array}{c}m \\ k\end{array}\right) x^{k}$ o sea $C_{m, o}^{(k)}=\left(\begin{array}{c}m \\ k\end{array}\right)$. Luego debemos probar que $\sum_{k=o}^{m}\left(\begin{array}{c}m \\ k\end{array}\right)^{2}=\frac{(2 m) !}{(m !)^{2}}$. Recordemos que $\left(\begin{array}{c}m \\ k\end{array}\right)=\left(\begin{array}{c}m \\ m-k\end{array}\right)$ y es consecuencia $(1+x)^{m}=\sum_{k=o}^{m}\left(\begin{array}{c}m \\ m-k\end{array}\right) x^{k}$, luego $(1+x)^{2 m}=\left[\sum_{k=o}^{m}\left(\begin{array}{c}m \\ k\end{array}\right) x^{k}\right]\left[\sum_{k=o}^{m}\left(\begin{array}{c}m \\ m-k\end{array}\right) x^{k}\right]$. De aquí vemos que el coeficiente de $x^{m}$ es $\left(\begin{array}{c}2 m \\ m\end{array}\right)=\sum_{k=o}^{m}\left(\begin{array}{c}m \\ k\end{array}\right)^{2}$ o sea $\sum_{k=o}^{m}\left(\begin{array}{c}m \\ k\end{array}\right)^{2}=\frac{(2 m) !}{(m !)^{2}}$

b) Debemos probar que si $A_{r}=\mathbb{N}$, entonces $A_{r+1}=\mathbb{N}$. Para ello observemos que:

$$
\begin{aligned}
& \sum_{k=0}^{m+1+r} C_{m+1, r}^{(k)} x^{k}=(1+x)^{m+1}(1-x)^{r}=\left(\sum_{k=o}^{m+r} C_{m, r}^{(k)} x^{k}\right)(1+x) \text { y que } \\
& \sum_{k=0}^{m+1+r} C_{m, r+1}^{(k)} x^{k}=(1+x)^{m}(1-x)^{r+1}=\left(\sum_{k=o}^{m+r} C_{m, r}^{(k)} x^{k}\right)(1-x) \text { luego: }
\end{aligned}
$$


$C_{m+1, r}^{(o)}=C_{m, r}^{(o)}=C_{m, r+1}^{(o)} ; C_{m+1, r}^{(m+r+1)}=C_{m, r}^{(m+r)}=-C_{m, r+1}^{(m+r+1)} ;$
$C_{m+1, r}^{(k)}=C_{m, r}^{(k)}+C_{m, r}^{(k-1)}$ y $C_{m, r+1}^{(k)}=C_{m, r}^{(k)}-C_{m, r}^{(k-1)} \forall k=1,2, \ldots, m+r$

Por 10 tanto:

$$
\begin{aligned}
& \sum_{k=0}^{m+r+1}\left(C_{m+1, r}^{(k)}\right)^{2}+\sum_{k=0}^{m+r+1}\left(C_{m, r+1}^{(k)}\right)^{2}=\left(C_{m, r}^{(o)}\right)^{2}+\sum_{k=1}^{m+r}\left(C_{m, r}^{(k)}+C_{m, r}^{(k-1)}\right)^{2}+\left(C_{m, r}^{(m+r)}\right)^{2}+ \\
& +\left(C_{m, r}^{(o)}\right)^{2}+\sum_{k-1}^{m+r}\left(C_{m, r}^{(k)}-C_{m, r}^{(k-1)}\right)^{2}+\left(C_{m, r}^{m+r}\right)^{2} \text { o sea } \\
& \sum_{k=0}^{m+r+1}\left(C_{m, r+1}^{(k)}\right)^{2}=4 \sum_{k=0}^{m+r}\left(C_{m, r}^{(k)}\right)^{2}-\sum_{k=0}^{m+r+1}\left(C_{m+1, r}^{(k)}\right)^{2} \text { pero por }
\end{aligned}
$$

Hipótesis de inducción sabemos que:

$$
\begin{aligned}
& \sum_{k=0}^{m+r}\left(C_{m, r}^{k}\right)^{2}=\frac{(2 m) !(2 r) !}{m ! r !(m+r) !} \forall \mathrm{m} \varepsilon \mathbb{N}, \quad \text { luego: } \\
& \sum_{k=0}^{m+r+1}\left(C_{m, r+1}^{(k)}\right)^{2}=\frac{4(2 m) !(2 r) !}{m ! r !(m+r) !}-\frac{(2 m+2) !(2 r) !}{(m+1) ! r !(m+r+1) !} \text { o sea } \\
& \sum_{k=0}^{m+r+1}\left(C_{m, r+1}^{(k)}\right)^{2}=\frac{(2 m) !(2[r+1]) !}{m !(r+1) !(m+r+1) !}
\end{aligned}
$$

